VLSI Design

1995, Vol. 2, No. 4, pp. 389-396

Reprints available directly from the publisher

Photocopying permitted by license only
(C) 1995 OPA (Overseas Publishers Association)

Amsterdam BV. Published under license by

Gordon and Breach Science Publishers SA

\title{
On Some Properties of the Star Graph
}

\author{
KE QIU \\ Command and Control Division, Defence Research Establishment Valcartier (DREV), 2459 Pie XI, Courcelette, PQ \\ Canada, G0A 1R0 \\ SELIM G. AKL \\ Department of Computing and Information Science, Queen's University, Kingston, Ontario, Canada, K7L 3N6
}

\begin{abstract}
We derive some properties of the star graph in this paper. In particular, we compute the number of nodes at distance $i$ from a fixed node $e$ in a star graph. To this end, a recursive formula is first obtained. This recursive formula is, in general, hard to solve for a closed form solution. We then study the relations among the number of nodes at distance $i$ to node $e$ in star graphs of different dimensions. This study reveals a very interesting relation among these numbers, which leads to a simple homogeneous linear recursive formula whose characteristic equation is easy to solve. Thus, we get a systematic way to obtain a closed form solution with given initial conditions for any fixed $i$.
\end{abstract}

Key Words: Star graph; Interconnection network; Topological property

\section{INTRODUCTION}

$\mathbf{L}$ et $V_{n}$ be the set of all $n$ ! permutations of symbols $1,2, \ldots, n$. For any permutation $v \in V_{n}$, if we denote the $i$ th symbol of $v$ by $v(i)$, then $v$ can be written as $v(1) v(2) \cdots v(n)$. We use the notation $i *$ to represent a permutation whose first symbol is $i$, where $*$ represents any permutation of the $n-1$ symbols in $\{1,2, \ldots, n\}-\{i\}$. Similarly, $* i$ represents a permutation whose last symbol is $i$. A star graph on $n$ symbols, $S_{n}=\left(V_{n}, E_{n}\right)$, is an undirected graph with $n$ ! nodes, where each node $v$ is connected to $n-1$ nodes which can be obtained by interchanging the first and $i$ th symbols of $v$, i.e., $(v(1) v(2) \cdots$ $v(i) v(i+1) \cdots v(n), v(i) v(2) \cdots v(i-1) v(1) v(i+1)$ $\cdots v(n)) \in E_{n}$, for $2 \leq i \leq n$. We call these $n-1$ connections dimensions. Thus each node is connected to $n-1$ nodes through dimensions 2, 3, $\ldots, n$. $S_{n}$ is also called an $n$-star or an $n$-dimensional star. Fig. 1 shows $S_{4}$.

The star graph is an attractive alternative to the hypercube, a popular network for interconnecting processors in a parallel computer, and compares favorably with it in several aspects $[1,2]$. For example, the degree of $S_{n}$ is $n-1$, i.e., sub-logarithmic in the number of nodes of $S_{n}$, while a hypercube with $\Theta(n !)$ nodes has degree $\Theta(\log n !)=\Theta(n \log n)$, i.e., log- arithmic in the number of nodes. The same can be said about the diameter of $S_{n}$. Much work has been done to study both the topological properties and parallel algorithms of the star graph lately $[3,4,7$, $11,13,14,15,16,17,18,19,20]$.

It is known that the star graph is both vertex symmetric and edge symmetric [1]. The vertex symmetry of the star graph implies that routing between two arbitrary nodes reduces to routing from an arbitrary node to the identity node $e=123 \cdots n$. Therefore, the diameter of $S_{n}, D(n)$, is the length of the longest shortest paths to $e$ among all the nodes in $S_{n}$, i.e. $D(n)=\max _{x \in V_{n}}\{d(x, e)\}$, where $d(x, y)$ denotes the shortest distance from node $x$ to node $y$.

In [2], a greedy algorithm is given which finds a path from any node $\pi$ to $e$. The algorithm is given by the following two rules:

1. if $\pi(1)=1$, move it to any position not occupied by the correct symbol, and

2. if $\pi(1)=x \neq 1$, move it to its correct position.

It is shown in [2] that this algorithm will always find the shortest path from $\pi$ to $e$ in $S_{n}$.

For a permutation $\pi$ of $n$ symbols, it is well known that $\pi$ has a unique cyclic representation (in canonical form) [12, page 176]. For example, the canonical cyclic representation of permutation $\pi=931846572$ 


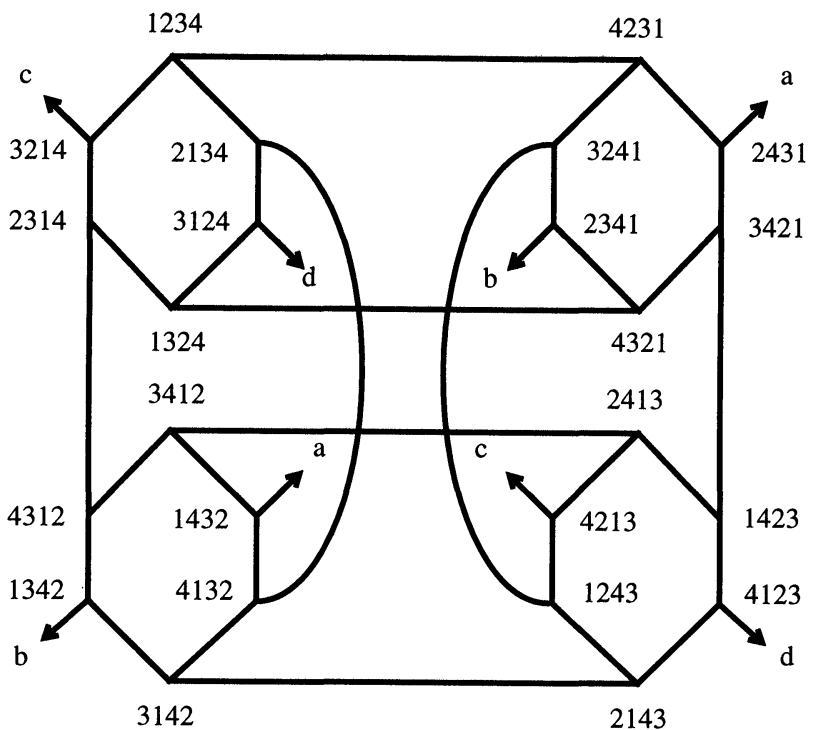

FIGURE 1 A 4-Star $S_{4}$.

is (6)(4875)(1923). Let $c$ be the number of cycles with more than one symbol, and $m$ the total number of symbols in these $c$ cycles, then the following theorem can be proved [2]:

Theorem 1 The shortest distance from $\pi$ to $e$ is given by:

$$
d(\pi, e)=c+m- \begin{cases}0 & \text { if } \pi(1)=1 \\ 2 & \text { otherwise }\end{cases}
$$

Therefore, $D(n)$ is the maximum value of $d(\pi, e)$ over all $n$ ! possible permutations. It has been shown that $D(n)$ is equal to $\lfloor 3(n-1) / 2\rfloor$ in $[1,2]$.

In this paper, we derive some properties of the star graph. In particular, we pose the following question: How many nodes are at distance $i$ from node $e$ in $S_{n}$ ? If the star graph is used as an interconnection network for interconnecting processors in a parallel computer such that each processor can communicate with all its neighbors in one time unit, then a direct application of our study is to obtain an answer to the following question: If a processor wants to send a message to as many processors as possible, then after $i$ steps, how many processors would have received the message?

Spanning trees of $S_{n}$ rooted at the identity $e$ have been proposed and studied in $[6,8,9]$. These spanning trees are similar to a breadth first spanning tree (BFST) rooted at $e$, in which the shortest distance from a node at level $i$ to $e$ is $i$. Note that a BFST rooted at any node in $S_{n}$ is not unique. However, the number of nodes at level $i$ of any BFST rooted at the same node is the same. Thus, the number of nodes at distance $i$ to $e$ in $S_{n}$ is the same as the number of nodes at level $i$ of a BFST of $S_{n}$ rooted at $e$. The motivation to study this spanning tree is similar to that of [10], which is to develop more efficient communication algorithms on the interconnection network. Therefore, our effort here is a first step in the study of the spanning tree structure of the star graphs.

Let $C_{k}^{m}=m ! /(k !(m-k) !)$ denote a binomial coefficient that represents the number of ways to choose $k$ objects from $m$ objects. For a hypercube of dimension $n$, the number of nodes at distance $i$ from node $000 \ldots 0$ can be easily obtained. Since the distance from node $a$ to $000 \cdots 0$ is equal to the total number of 1's in $a$ 's binary representation, namely the Hamming distance of $a$, it follows that the total number is $C_{i}^{n}$.

The problem of computing the number of nodes at a given distance from an origin is also studied for rotator graphs in [5]. This distance is used to find the average distance between nodes in a rotator graph (the average distance in a star graph is obtained through a different method in [2]).

In an $n$-star, one way to compute this number is to find the number of permutations $\pi$ satisfying:

$$
i=c+m- \begin{cases}0 & \text { if } \pi(1)=1 \\ 2 & \text { otherwise }\end{cases}
$$

This task seems difficult in general, except for some specific $i$ 's, for example, when $i$ is small or when $i$ is the diameter. For example, when $i=1$, the only choice is that $c=1$, and $m=2$, and the permutations are

$$
\begin{array}{llll}
2134 & \cdots & n \\
3214 & \cdots & n \\
\vdots & & \\
n 234 & \cdots & 1
\end{array}
$$

Also, when $i$ is the diameter, it is easy to compute the total number of nodes whose distance to $e$ is $i$. For example, when $n=2 k+1$ is an odd number, then all the nodes whose distance to $e$ is $D(n)$ $(=3 k)$ are of the form $1 *[20$, Lemma 1$)$. In this case, it is easy to show that the total number of nodes at distance $D(n)$ is $1 \times 3 \times 5 \times \cdots \times(2 k-1)$.

In the next section, we first derive a recursive formula which computes the total number of nodes at distance $i$ from node $e$ in $S_{n}$. However, it is worth pointing out that the recursive formula obtained has two parameters, and is hard to solve in general. The 
approach used is to count the number of nodes of a specific form at a particular distance, and the total number of nodes at that distance is simply the sum of all these numbers. We then continue our study in Section 3, using a different approach, to obtain a nice recursive formula for the total number of nodes at distance $i$ from $e$ in $S_{n}$. This recursive formula is simple and its characteristic equation is easy to solve. Therefore, given initial conditions, there is a systematic way to obtain a closed form solution to the recursive equation for any fixed $i$.

\section{COUNTING THE NUMBER OF NODES AT DISTANCE $i$ FROM $e:$ I}

Let $S_{n-1}(i)$ be a sub-graph of $S_{n}$ induced by all the nodes of the form $* i$, for some $1 \leq i \leq n$. It can be seen that $S_{n-1}(i)$ is an $(n-1)$-star defined on symbols $\{1,2, \cdots, n\}-\{i\}$. Thus, $S_{n}$ can be decomposed into $n S_{n-1}$ 's: $S_{n-1}(i), 1 \leq i \leq n[1,2]$. For example, $S_{4}$ in Fig. 1 contains four 3-stars, namely $S_{3}(1)$, $S_{3}(2), S_{3}(3)$, and $S_{3}(4)$, respectively.

We use $f(n, i, j)$ to denote the number of nodes of the form $j *$ at distance $i$ from node $e$ in $S_{n}$, and we use $g(n, i)$ to denote the total number of nodes at distance $i$ from $e$ in $S_{n}$, for $1 \leq j \leq n$ and $0 \leq i$ $\leq\lfloor 3(n-1) / 2\rfloor$. Clearly,

$$
\begin{gathered}
g(n, i)=\sum_{j=1}^{n} f(n, i, j), \\
\sum_{i=0}^{\lfloor 3(n-1) / 2\rfloor} g(n, i)=n ! .
\end{gathered}
$$

It is easily seen that:

- $g(n, 0)=f(n, 0,1)=1$, and $f(n, 0, j)=0$ for $2 \leq j \leq n$

- $g(n, 1)=n-1, f(n, 1, j)=1$ for $2 \leq j \leq n$, and $f(n, 1,1)=0$;

- $g(n, 2)=(n-2)(n-1), f(n, 2, j)=n-2$ for $2 \leq j \leq n$, and $f(n, 2,1)=0$.

The following lemma immediately follows from the edge-symmetry of the star graph.

Lemma $1 f(n, i, j)=f(n, i, k)$, for $2 \leq j, k \leq n$.

Lemma 2 For $2 \leq j \leq n+1$ and $0 \leq i \leq \mathrm{L} 3(n-$ 1)/2」, we have $f(n+1, i+1, j)=g(n$, i), i.e. the number of nodes of the form $j *$, where $2 \leq j \leq n+$ 1 , at distance $i+1$ from $e$ in $S_{n+1}$ is equal to the total number of nodes at distance $i$ from $e$ in $\mathrm{S}_{n}$.

$$
\begin{gathered}
11 * \\
12 *, 13 *, 14 * \\
22 *, 23 *, 24 * \\
31 *, 22 *, 23 *, 24 * \\
21 *, 12 *, 13 *, 14 *
\end{gathered}
$$

FIGURE 2 Nodes at different distances from $e$ in $S_{4}$.

Proof: By Lemma 1, we only need to show that the lemma is true for $j=n+1$.

By the greedy algorithm, if there exist shortest paths of length $i+1$ from node of the form $(n+$ $1) *$ to $12 \cdots n(n+1)$, then at least one of such paths takes the form of

$$
(n+1) * \rightarrow \underbrace{*(n+1) \rightarrow \cdots \rightarrow 123 \cdots n(n+1)}_{i \text { steps, all inside } S_{n}(n+1)}
$$

Therefore, the number of nodes of the form $(n+1) *$ at distance $i+1$ from node $123 \cdots n(n+1)$ is equal to the number of nodes of the form $*(n+1)$ at distance $i$ from node $123 \cdots n(n+1)$, which is $g(n$, i). Thus it follows that $f(n+1, i+1, n+1)=g(n$, i). Edge symmetry of the star graph implies that $f(n$ $+1, i+1, j)=g(n, i), 2 \leq j \leq n+1$.

The numbers of nodes of different forms at different distances in $S_{4}, S_{5}$, and $S_{6}$ are given in italics in Figures 2, 3, and 4. Lemma 2 says that the sequence of $j$ 's $(j \neq 1)$ coefficients at different distances in $S_{n+1}$ is the same as the sequence of total number of nodes at different distances in $S_{n}$. For example, the sequence for $2 *$ 's in $S_{6}$ is $1,4,12,30,44$, 26,3 , the same as the sequence for $g(5, i), 0 \leq i \leq$ 6 (see Table I). By checking the diameter of $S_{n}$, given by $\lfloor 3(n-1) / 2\rfloor$, we notice that $D(n+1)=D(n)+$ 2 when $n$ is even, but nodes whose distance to $e$ is $D(n+1)$ are all of the form $1 *$. When $n$ is odd, $D(n$ $+1)=D(n)+1$. Therefore, Lemma 2 gives a way to compute the number of nodes of the form $j *, 2 \leq$ $j \leq n$, at any distance from $e$ in $S_{n}$, as a function of $g(n-1, i-1)$.

$$
\begin{gathered}
11 * \\
12 *, 13 *, 14 *, 15 * \\
32 *, 33 *, 34 *, 35 * \\
61 *, 62 *, 63 *, 64 *, 65 * \\
81 *, 92 *, 93 *, 94 *, 95 * \\
61 *, 52 *, 53 *, 54 *, 55 * \\
31 *
\end{gathered}
$$

FIGURE 3 Nodes at different distances from $e$ in $S_{5}$. 


$$
\begin{gathered}
11 * \\
12 *, 13 *, 14 *, 15 *, 16 * \\
42 *, 43 *, 44 *, 45 *, 46 * \\
101 *, 122 *, 123 *, 124 *, 125 *, 126 * \\
201 *, 302 *, 303 *, 304 *, 305 *, 306 * \\
301 *, 442 *, 443 *, 444 *, 445 *, 446 * \\
391 *, 262 *, 263 *, 264 *, 265 *, 266 * \\
201 *, 32 *, 33 *, 34 *, 35 *, 36 *
\end{gathered}
$$

FIGURE 4 Nodes at different distances from $e$ in $S_{6}$.

Lemma 3 For $2 \leq j \leq n$, we have $f(n+1, i, 1)$ $=f(n, i, 1)+(n-1) f(n, i-2, j)$.

Proof: By the hierarchical structure of the star graph, $S_{n+1}$ can be partitioned into $n+1 n$-stars $S_{n}(n$ $+1), S_{n}(n), \ldots, S_{n}(2), S_{n}(1)$. Clearly, there is no node of the form $1 *$ in sub-star $S_{n}(1)$. Let the number of nodes of the form $1 * k$ in $S_{n}(k)$ at distance $i$ to $e$ in $S_{n+1}$ be $N(k, i)$, for $2 \leq k \leq n+1$, then $f(n+$ $1, i, 1)=\sum_{k=2}^{n+1} N(k, i)$. Clearly, $N(n+1, i)=$ $f(n, i, 1)$.

By edge symmetry, $N(k, i)=N(l, i)$, for $2 \leq k, l$ $\leq n$. We now show that $N(k, i)=f(n, i-2, k)$, for $2 \leq k \leq n$. For each node of the form $1 * k$ at distance $i$ to $e$, by the greedy algorithm, one optimal path is as follows:

$$
1 * k \rightarrow \underbrace{k * 1 \rightarrow \cdots \rightarrow 12 \cdots n(n+1)}_{i-1 \text { steps }}
$$

Now consider this $k * 1$ as the identity (by vertex symmetry) and apply the greedy algorithm to find a shortest path from node $12 \cdots n(n+1)$ to the node $k * 1$. In this path, we would go as follows:

$$
12 \cdots n(n+1) \rightarrow \underbrace{(n+1) 23 \cdots n 1 \rightarrow \cdots \rightarrow k * 1}_{i-2 \text { steps }}
$$

so the path length from node $k * 1$ to $(n+1) 23 \ldots$ $n 1$ is $i-2$. Therefore, all nodes of the form $1 * k$ whose distances to $123 \cdots n(n+1)$ is $i$ will have a path

$$
\begin{aligned}
& 1 * k \rightarrow \\
& \underbrace{k * 1 \rightarrow \text { steps }}_{i-2}
\end{aligned}
$$

So the total number of such nodes is equal to the total number of nodes of the form $k * 1$ whose distance to $(n+1) 23 \cdots n 1$ is $i-2$, which, in turn, is $f(n, i-2, k)$ by vertex symmetry (by considering node $(n+1) 23 \cdots n 1$ as the identity in $\left.S_{n}(1)\right)$.

Combining the above results, we have

$$
\begin{aligned}
& f(n+1, i, 1) \\
& \quad=f(n, i, 1)+(n-1) f(n, i-2, j), \quad 2 \leq j \leq n .
\end{aligned}
$$

Theorem 2 In $S_{n}$, the total number of nodes at distance $i$ from e can be computed recursively as follows: For $n \geq 1$ and $i \leq 2$,

$$
\begin{aligned}
& g(n, 0)=1 \\
& g(n, 1)=n-1 \\
& g(n, 2)=(n-1)(n-2)
\end{aligned}
$$

\begin{tabular}{|c|c|c|c|c|c|c|c|c|c|c|c|}
\hline & $i=0$ & $i=1$ & $i=2$ & $i=3$ & $i=4$ & $i=5$ & $i=6$ & $i=7$ & $i=8$ & $i=9$ & $i=10$ \\
\hline$n=1$ & 1 & & & & & & & & & & \\
\hline$n=2$ & 1 & 1 & & & & & & & & & \\
\hline$n=3$ & 1 & 2 & 2 & 1 & & & & & & & \\
\hline$n=4$ & 1 & 3 & 6 & 9 & 5 & & & & & & \\
\hline$n=5$ & 1 & 4 & 12 & 30 & 44 & 26 & 3 & & & & \\
\hline$n=6$ & 1 & 5 & 20 & 70 & 170 & 250 & 169 & 35 & & & \\
\hline$n=7$ & 1 & 6 & 30 & 135 & 460 & 1110 & 1689 & 1254 & 340 & 15 & \\
\hline$n=8$ & 1 & 7 & 42 & 231 & 1015 & 3430 & 8379 & 13083 & 10408 & 3409 & 315 \\
\hline
\end{tabular}

and for $n \geq 1$ and $3 \leq i \leq D(n)$,

$$
\begin{aligned}
g(n, i)= & (n-1) g(n-1, i-1) \\
& +\sum_{j=1}^{n-2} j g(j, i-3) .
\end{aligned}
$$

Proof: The theorem follows directly from Lemmas 2, 3, and Eq. 1.

From the theorem, some properties about the number of nodes at different distances from $e$ of a star graph can be derived easily as follows:

Corollary 1 (1). For $i \leq n-2, g(n, i) \leq g(n, i+$ $1)$, and the equality holds only when $n=2$ and $n=$ 3 for some $i$ 's; and (2). For $i \geq n-1, g(n, i)>$ $g(n, i+1)$. That is, for any fixed $n$, the function

TABLE I

Total Number of Nodes at Different Distances From $e$ in $S_{n}, n \leq 8$ 
$g(n, i)$ increases as $i$ increases until it reaches its maximum $g(n, n-1)$ at $i=n-1$, then decreases as $i$ increases after $n-1$.

Proof: By simple induction on $n$.

For $n=1,2,3$, and 4 , the claim is true by checking Table I. Note that the equality holds only when $n=2$ or 3 for some $i$ 's.

Assume that the claim is true for $n>4$, then for $n+1$, we need to show that (a) $g(n+1, i) \leq g(n$ $+1, i+1)$ for $i \leq(n+1)-2=n-1$, and (b) $g(n+1, i)>g(n+1, i+1)$ for $i \geq(n+1)-1$.

From Theorem 2:

$$
\begin{aligned}
& g(n+1, i)=n g(n, i-1)+\sum_{j=1}^{n-1} j g(j, i-3) \\
& g(n+1, i+1)=n g(n, i)+\sum_{j=1}^{n-1} j g(j, i-3)
\end{aligned}
$$

By our induction hypothesis, we know that $g(n, i-$ 1) $<\cdot g(n, i)$ for $i \leq n-1$, thus $g(n+1, i)<$ $g(n+1, i+1)$ for $i \leq(n+1)-2=n-1$. Also, for $i \geq n, g(n, i-1)>g(n, i)$ by induction hypothesis, thus $g(n+1, i)>g(n+1, i+1)$ for $i \geq n$.

This recursive formula (Eq. 2) has two parameters, and has non-constant coefficients, which make it hard to obtain a closed form solution in general. In the next section, we will study the problem from a different angle to get a systematic way of obtaining. a closed form solution to the problem.

Table I gives the number of nodes at different distances from $e$ in $S_{n}$ for $n \leq 8$.

\section{COUNTING THE NUMBER OF NODES AT DISTANCE $i$ FROM $e$ : II}

Our purpose is to derive a recursive equation to which a closed form solution can be easily obtained. To this end, we now check each column in Table I closely to see whether there is any correlation among the number of nodes at the same distance for stars of different dimensions.

From Table I, we observe that:

$$
\begin{aligned}
& g(n+1,0)-g(n, 0)=0 \text { for } n \geq 1 \\
& g(n+2,1)-2 g(n+1,1)+g(n, 1) \\
& \quad=0 \text { for } n \geq 2
\end{aligned}
$$

$$
\begin{aligned}
& g(n+3,2)-3 g(n+2,2)+3 g(n+1,2) \\
& \quad-g(n, 2)=0 \text { for } n \geq 3 \\
& g(n+4,3)-4 g(n+3,3)+6 g(n+2,3) \\
& \quad-4 g(n+1,3)+g(n, 3)=0 \text { for } n \geq 3 \\
& g(n+5,4)-5 g(n+4,4)+10 g(n+3,4) \\
& \quad-10 g(n+2,4)+5 g(n+1,4)-g(n, 4) \\
& \quad=0 \text { for } n \geq 4
\end{aligned}
$$

Of course, the above observation is valid only for the entries in that table. But in fact, we can show that the above observation can be generalized as follows:

Theorem 3 For any $i, n$, such that $0 \leq i \leq$ $\lfloor 3(n-1) / 2\rfloor$, we have:

$$
\begin{aligned}
\sum_{k=0}^{i+1}( & -1)^{k} C_{k}^{i+1} g(n+i+1-k, i) \\
= & g(n+i+1, i)-C_{1}^{i+1} g(n+i, i) \\
& +C_{2}^{i+1} g(n+i-1, i)-\cdots \\
& +(-1)^{i+1} C_{i+1}^{i+1} g(n, i) \\
= & 0
\end{aligned}
$$

Proof: We prove the theorem by applying induction on $i$.

From Theorem 2 we know that:

- for $i=0$, and $n \geq 1$,

$$
g(n+1,0)-g(n, 0)=1-1=0 ;
$$

- for $i=1$, and $n \geq 2$,

$$
\begin{aligned}
& g(n+2,1)-2 g(n+1,1)+g(n, 1) \\
& \quad=(n+1)-2 n+(n-1) \\
& \quad=0
\end{aligned}
$$

- for $i=2$, and $n \geq 3$,

$$
\begin{aligned}
g(n+ & +3,2)-3 g(n+2,2)+3 g(n+1,2) \\
& -g(n, 2)=(n+2)(n+1)-3(n+1) n \\
& +3 n(n-1)-(n-1)(n-2) \\
= & 0 .
\end{aligned}
$$

Assume that the theorem is true for all $h \leq i$, where $i \geq 3$, i.e. 


$$
\begin{aligned}
\sum_{k=0}^{h+1}( & -1)^{k} C_{k}^{h+1} g(n+h+1-k, h) \\
= & g(n+h+1, h)-C_{1}^{h+1} g(n+h, h) \\
& +C_{2}^{h+1} g(n+h-1, h)-\cdots \\
& +(-1)^{h+1} C_{h+1}^{h+1} g(n, h) \\
= & 0,
\end{aligned}
$$

for $h \leq\lfloor 3(n-1) / 2\rfloor$. We now need to show that for $i+1 \leq\lfloor 3(n-1) / 2\rfloor$,

$$
\begin{aligned}
\mathcal{F}= & \sum_{k=0}^{i+2}(-1)^{k} C_{k}^{i+2} g(n+i+2-k, i+1) \\
= & g(n+i+2, i+1) \\
& -C_{1}^{i+2} g(n+i+1, i+1) \\
& +C_{2}^{i+2} g(n+i, i+1)+\cdots \\
& +(-1)^{i+2} C_{i+2}^{i+2} g(n, i+1) \\
= & 0 .
\end{aligned}
$$

By Theorem 2, we know that

$$
\begin{aligned}
& g(n+i+2, i+1)-C_{1}^{i+2} g(n+i+1, i+1) \\
& \quad+C_{2}^{i+2} g(n+i, i+1)+\cdots \\
& \quad+(-1)^{i+2} C_{i+2}^{i+2} g(n, i+1) \\
& =\left[(n+i+1) g(n+i+1, i)+\sum_{j=1}^{n+i} j g(j, i-2)\right] \\
& \quad-C_{1}^{i+2}\left[(n+i) g(n+i, i)+\sum_{j=1}^{n+i-1} j g(j, i-2)\right] \\
& \quad+C_{2}^{i+2}[(n+i-1) g(n+i-1, i) \\
& \left.\quad+\sum_{j=1}^{n+i-2} j g(j, i-2)\right] \\
& \quad \vdots \\
& \quad+(-1)^{i+2} C_{i+2}^{i+2}[(n-1) g(n-1, i) \\
& \left.\quad+\sum_{j=1}^{n-2} j g(j, i-2)\right]
\end{aligned}
$$

$$
\begin{aligned}
= & (n+i+1) g(n+i+1, i) \\
& -C_{1}^{i+2}(n+i) g(n+i, i)+\cdots \\
& +(-1)^{i+2} C_{i+2}^{i+2}(n-1) g(n-1, i) \\
& +\sum_{j=1}^{n+i} j g(j, i-2)-C_{1}^{i+2} \sum_{j=1}^{n+i-1} j g(j, i-2) \\
& +C_{2}^{i+2} \sum_{j=1}^{n+i-2} j g(j, i-2)+\cdots \\
& +(-1)^{i+2} C_{i+2}^{i+2} \sum_{j=1}^{n-2} j g(j, i-2) \\
= & \sum_{k=0}^{i+2}(-1)^{k} C_{k}^{i+2}(n+i+1-k) g(n+i+1-k, i) \\
& +\sum_{k=0}^{i+2}(-1)^{k} C_{k}^{i+2}\left(\sum_{j=1}^{n+i-k} j g(j, i-2)\right) \\
= & F_{1}+\mathcal{F}_{2},
\end{aligned}
$$

where

$$
\begin{aligned}
\mathcal{F}_{1}= & \sum_{k=0}^{i+2}(-1)^{k} C_{k}^{i+2}(n+i+1-k) g(n+i \\
& +1-k, i) \\
\mathcal{F}_{2}= & \sum_{k=0}^{i+2}(-1)^{k} C_{k}^{i+2}\left(\sum_{j=1}^{n+i-k} j g(j, i-2)\right) .
\end{aligned}
$$

Let

$$
\begin{aligned}
& \alpha=\sum_{k=0}^{i+1}(-1)^{k} C_{k}^{i+1} g(n+i+1-k, i), \\
& \beta=\sum_{k=0}^{i+1}(-1)^{k} C_{k}^{i+1} g(n+i-k, i), \\
& \mathfrak{Q}=\sum_{k=0}^{i-1}(-1)^{k} C_{k}^{i-1} g(n+i-k, i-2), \\
& \mathcal{B}=\sum_{k=0}^{i-1}(-1)^{k} C_{k}^{i-1} g(n+i-1-k, i-2), \\
& \mathcal{C}=\sum_{k=0}^{i-1}(-1)^{k} C_{k}^{i-1} g(n+i-2-k, i-2) .
\end{aligned}
$$

From the induction hypothesis, we know that $\alpha=\beta$ $=\mathfrak{A}=\mathfrak{B}=\mathfrak{C}=0$.

We now show $\mathfrak{F}=0$ by showing that 


$$
\begin{aligned}
\mathcal{F}= & (n+i+1) \alpha-(n-1) \beta+(n+i) \mathfrak{Q} \\
& +(-2 n-i+1) \mathfrak{B}+(n-1) \mathfrak{C} .
\end{aligned}
$$

To this end, we show that

$\mathfrak{F}_{1}=(n+i+1) \alpha-(n-1) \beta$,

$\mathcal{F}_{2}=(n+i) \mathfrak{Q}+(-2 n-i+1) \mathscr{B}+(n-1) \mathcal{C}$

To show that Eq. 4 holds, we compare the coefficients of function $g(m, i)$, for $n-1 \leq m \leq n+i$ +1 , i.e., for $0 \leq k \leq i+2$, on both sides of the equation. To show that Eq. 5 holds, we compare the coefficients of the corresponding $g(j, i-2)$ 's on both sides of the equation for $1 \leq j \leq n+i$. The proof is then straightforward and can be found in [17].

Note that although Eq. 3 has two parameters, it actually has only one for any fixed value of $i$. Thus we are left with a linear homogeneous recursive equation with constant coefficients, which can be solved easily as follows:

The characteristic equation of Eq. 3 is:

$$
(x-1)^{i+1}=0
$$

where $x=1$ is the only root and it is a multiple root. Therefore, the solution to Eq. 3 takes the form:

$$
g(n, i)=c_{1}+c_{2} n+c_{3} n^{2}+\cdots+c_{i+1} n^{i}
$$

where $c_{j}$ 's, $1 \leq j \leq i+1$, are constants to be determined later. This gives us an estimate about the value of $g(n, i)$, namely, a polynomial in $n$ of degree $i$.

Eq. 6 gives a closed form solution for the total number of nodes at distance $i$ from the identity $e$ for any $n$, provided that $0 \leq i \leq\lfloor 3(n-1) / 2\rfloor$. The $i+$ 1 constants $c_{j}$ can be determined by initial conditions to the recurrence Eq. 3, which are $i+1$ values $g\left(n_{1}\right.$, $i), g\left(n_{2}, i\right), \ldots, g\left(n_{i+1}, i\right)$, where $i \leq\left\lfloor 3\left(n_{j}-1\right) / 2\right\rfloor$, for $1 \leq j \leq i+1$. Initial conditions can be computed by using the result of Theorem 2 . Substituting these values into Eq. 6, we get a system of linear equations:

$$
\left\{\begin{array}{l}
c_{1}+c_{2} n_{1}+c_{3} n_{1}^{2}+\cdots+c_{i+1} n_{1}^{i}=g\left(n_{1}, i\right) \\
c_{1}+c_{2} n_{2}+c_{3} n_{2}^{2}+\cdots+c_{i+1} n_{2}^{i}=g\left(n_{2}, i\right) \\
\vdots \\
c_{1}+c_{2} n_{i}+c_{3} n_{i}^{2}+\cdots+c_{i+1} n_{i}^{i}=g\left(n_{i}, i\right) \\
c_{1}+c_{2} n_{i+1}+c_{3} n_{i+1}^{2}+\cdots+c_{i+1} n_{i+1}^{i}=g\left(n_{i+1}, i\right) .
\end{array}\right.
$$

The coefficient matrix of the system is

$$
\left(\begin{array}{ccccc}
1 & n_{1} & n_{1}^{2} & \cdots & n_{1}^{i} \\
1 & n_{2} & n_{2}^{2} & \cdots & n_{2}^{i} \\
\vdots & \vdots & \vdots & \vdots & \vdots \\
1 & n_{i+1} & n_{i+1}^{2} & \cdots & n_{i+1}^{i}
\end{array}\right)
$$

This is the transpose of a Vandermonde matrix [12, page 36] whose determinant is known to be $\Pi_{1 \leq j<k \leq i+1}\left(n_{k}-n_{j}\right)$. This determinant is not equal to 0 unless there exist $k$ and $j$ such that $1 \leq j, k \leq i+$ $1, k \neq j$, and $n_{k}=n_{j}$. Therefore, the system of linear equations has a unique solution when $i+1$ distinct initial values $g\left(n_{1}, i\right), g\left(n_{2}, i\right), \ldots, g\left(n_{i+1}, i\right)$, are given such that $n_{k} \neq n_{j}$ if $k \neq j$, and $1 \leq k, j \leq$ $i+1$.

We now give an example for $i=4$. The general solution is:

$$
g(n, 4)=c_{1}+c_{2} n+c_{3} n^{2}+c_{4} n^{3}+c_{5} n^{4},
$$

for $n \geq 4$. The initial conditions are:

$$
\begin{aligned}
& g(4,4)=5, \\
& g(5,4)=44, \\
& g(6,4)=170, \\
& g(7,4)=460, \\
& g(8,4)=1015 .
\end{aligned}
$$

The system of linear equations is:

$$
\left\{\begin{array}{l}
c_{1}+4 c_{2}+16 c_{3}+64 c_{4}+256 c_{5}=5 \\
c_{1}+5 c_{2}+25 c_{3}+125 c_{4}+725 c_{5}=44 \\
c_{1}+6 c_{2}+36 c_{3}+216 c_{4}+1296 c_{5}=170 \\
c_{1}+7 c_{2}+49 c_{3}+343 c_{4}+2401 c_{5}=460 \\
c_{1}+8 c_{2}+64 c_{3}+512 c_{4}+4096 c_{5}=1015
\end{array}\right.
$$

Solving this, we get:

$$
\begin{aligned}
& c_{1}=19, \\
& c_{2}=-245 / 6, \\
& c_{3}=30, \\
& c_{4}=-55 / 6, \\
& c_{5}=1 .
\end{aligned}
$$

Therefore, 


$$
g(n, 4)=19-245 n / 6+30 n^{2}-55 n^{3} / 6+n^{4} .
$$

Thus, for $n=10$, say, $g(10,4)=3444$.

\section{CONCLUSION}

In this paper, we derived some properties of the star graph. In particular, we obtained a recursive formula to compute the number of nodes at any distance to a fixed node in $S_{n}$. We also presented a systematic way for obtaining a closed form solution that gives the number of nodes at distance $i$ from $e$ for any fixed $i$. These properties are interesting in their own right. This study will help us better understand star graphs. Finally, it is worth noting that although our approach gives a systematic way to compute a closed form expression for $g(n, i)$, for any fixed $i$ and any $n$, it does need $i+1$ initial conditions (which can be obtained by using recursive equation Eq. 2). Nevertheless, the result of Section 3 characterizes the behavior of the number of nodes at any distance from $e$ in $S_{n}$. It remains an open problem to obtain a closed form solution for $g(n, i)$ for any fixed $n$ and any $i, i \leq\lfloor 3(n-1) / 2\rfloor$, without using any initial conditions.

\section{Acknowledgment}

We thank the anonymous referees for their helpful comments.

\section{References}

[1] S.B. Akers and B. Krishnamurthy, "A Group Theoretic Model for Symmetric Interconnection Networks," IEEE Trans. on Compu., Vol. c-38, No. 4, 1989, pp. 555-566.

[2] S.B. Akers, D. Harel, and B. Krishnamurthy, "The Star Graph: An Attractive Alternative to the $n$-cube," Proc. International Conference on Parallel Processing, St. Charles, Illinois, 1987, pp. 393-400.

[3] S.G. Akl and K. Qiu, "A Novel Routing Scheme on the Star and Pancake Networks and Its Applications," Parallel Computing, Vol. 19, No. 1, 1993, pp. 95-101.

[4] S.G. Akl, K. Qiu, and I. Stojmenović, "Fundamental Algorithms for the Star and Pancake Interconnection Networks with Applications to Computational Geometry," Networks: An International Journal, Special issue on Interconnection Networks and Algorithms, Vol. 23, 1993, pp. 215-225.

[5] P.F. Corbett, "Rotator Graphs: An Efficient Topology for Point-to-Point Multiprocessor Networks," IEEE Trans. on Parallel and Distributed Systems, Vol. 3, No. 5, Sept. 1992, pp. 622-626.

[6] K. Day and A. Tripathi, "A Comparative Study of Topological Properties of Hypercube and Star Graphs," to appear in IEEE Trans. on Parallel and Distributed Systems.

[7] K. Day and A. Tripathi, "Unidirectional Star Graphs," Information Processing Letters, 45, 1993, pp. 123-129.

[8] P. Fragopoulou and S.G. Akl, "Optimal Communication Algorithms on Star Graphs Using Spanning Tree Construc- tions," to appear in Proc. of the 5th IEEE Symp. on Parallel and Distributed Processing, Texas, Dec. 1993.

[9] P. Fragopoulou and S.G. Akl, Multiple Edge-Disjoint Spanning Trees on the Star Interconnection Network with Applications to Fault Tolerance, Technical Report No. 93-353, Department of Computing and Information Science, Queen's University, Kingston, Canada, 1993.

[10] C.T. Ho and S.L. Johnsson, "Distributed Routing Algorithms for Broadcasting and Personalized Communication in Hypercube," Proc. International Conference on Parallel Processing, St. Charles, Illinois, 1986, pp. 640-648.

[11] J.S. Jwo, S. Lakshmivarahan, and S.K. Dhall, "Embedding of Cycles and Grids in Star Graphs," Proc. 2nd IEEE Symp. Parallel and Distributed Processing, Dallas, Texas, 1990, pp. 540-547.

[12] D.E. Knuth, The Art of Computer Programming, Vol. 1, Addison-Wesley, Reading, Massachusetts, 1973.

[13] A. Menn and A.K. Somani, "An Efficient Sorting Algorithm for the Star Graph Interconnection Network," Proc. International Conference on Parallel Processing, St. Charles, Illinois, 1990, pp. 1-8.

[14] M. Nigam, S. Sahni, and B. Krishnamurthy, "Embedding Hamiltonians and Hypercubes in Star Interconnection Graphs," Proc. International Conference on Parallel Processing, St. Charles, Illinois, 1990, pp. 340-343.

[15] K. Qiu and S.G. Akl, "Load Balancing, Selection, and Sorting on the Star and Pancake Networks," to appear in Journal of Parallel Algorithms and Applications.

[16] K. Qiu, S.G. Akl, and H. Meijer, "On Some Properties and Algorithms for the Star and Pancake Interconnection Networks," to appear in Journal of Parallel and Distributed Computing.

[17] K. Qiu and S.G. Akl, Properties of the Breadth First Spanning Tree of the Star Graph, Technical Report 93-350, Department of Computing and Information Science, Queen's University, Kingston, Ontario, Canada, 1993.

[18] X. Shen, Q. Hu, B. Cong, H. Sudborough, M. Girou, and S. Bettayeb, "The 4-Star Graph Is Not a Subgraph of Any Hypercube," Information Processing Letters, 45, 1993, pp. 199-203.

[19] P.K. Srimani, Generalized Fault Tolerance Properties of Star Graphs, Technical Report CS-90-104, Department of Computer Science, Colorado State University, 1990.

[20] S. Sur and P.K. Srimani, "A Fault Tolerant Routing Algorithm in Star Graphs," Proc. International Conference on Parallel Processing, Vol. III, St. Charles, Illinois, August 1991, pp. 267-270.

\section{Biographies}

KE QIU received a B.Sc. in Computer Science from Harbin Institute of Technology, China, an M.Sc. in Computer Science, and an M.Sc. in Applied Mathematics, both from University of California at Davis, and a Ph.D. in Computer Science from Queen's University, Kingston, Canada. His major research interest is in the area of parallel algorithms and interconnection networks. Currently, he is with Defence Research Establishment Valcartier, Quebec, Canada.

SELIM G. AKL received his Ph.D. from McGill University in 1978, and is currently a Professor at Queen's University. His research interests are in the area of parallel computation. He is author of Parallel Sorting Algorithms (Academic Press, 1985), The Design and Analysis of Parallel Algorithms (Prentice Hall, 1989), and co-author of Parallel Computational Geometry (Prentice Hall, 1993). He is an editor of Information Processing Letters, Computational Geometry, Parallel Processing Letters, and the Journal of Parallel Algorithms and Applications, and a member of IEEE and ACM. In 1990 Dr. Akl occupied the Louis Néel Chair at the Laboratoire de l'Informatique du Parallélisme of the Ecole Normale Supérieure de Lyon, France. 

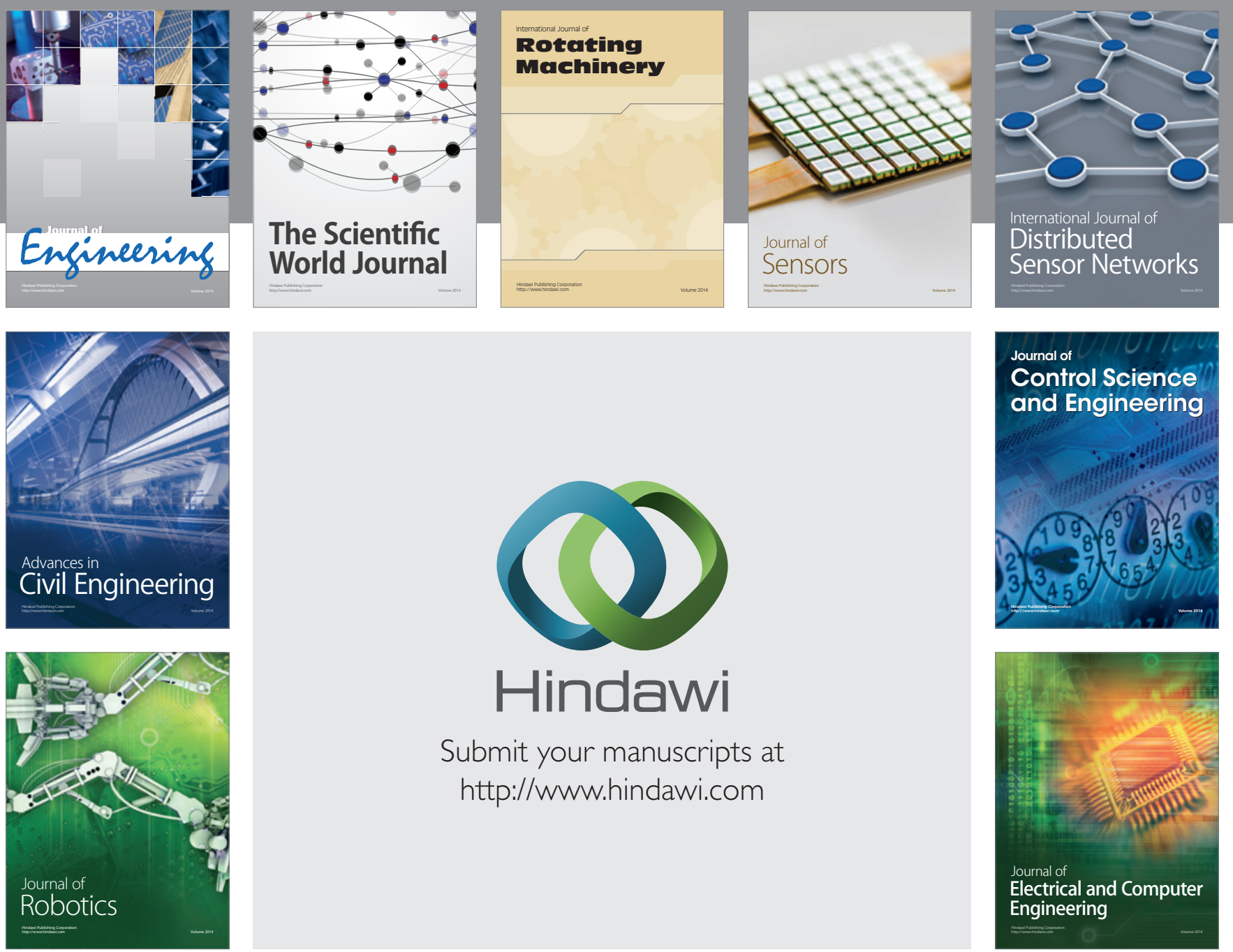

Submit your manuscripts at

http://www.hindawi.com
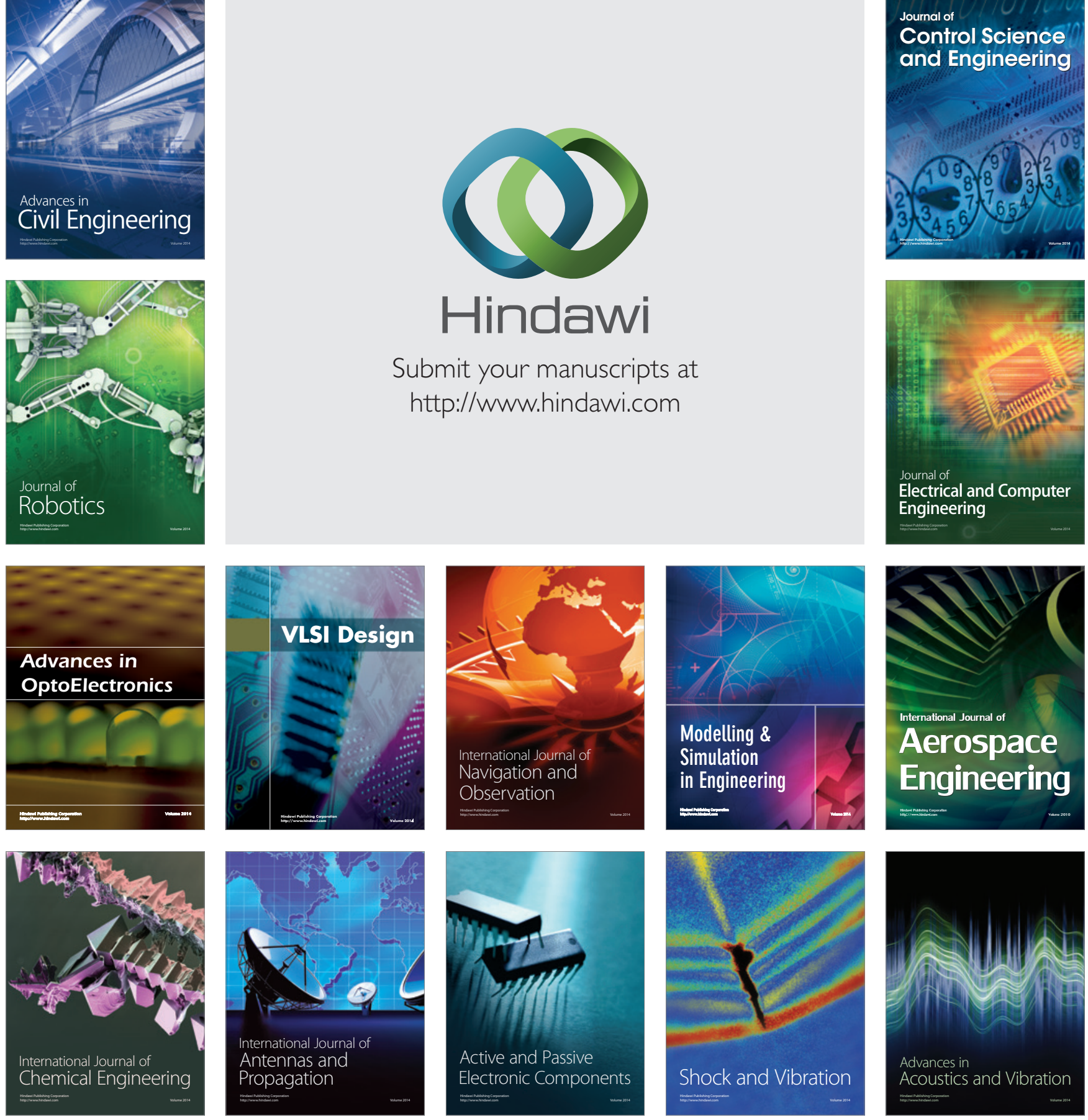